\title{
Des communes suisses en réseau \\ La référence à la montagne dans les partenariats à distance noués par les acteurs locaux
}

\section{Bernard Debarbieux, Gilles Rudaz, Genève}

\section{L'internationalisation des enjeux montagnards et la place des populations locales}

Depuis une quinzaine d'années, la montagne tend à devenir un objet de préoccupations intergouvernementales et transnationales (Messerli \& Hofer 2003). Entre la Conférence des Nations Unies sur l'Environnement et le Développement (1992), qui a conduit à l'inscription dans l'Agenda 21 d'un chapitre spécifique dédié aux écosystèmes de montagne, et la proclamation d'une Année Internationale de la Montagne (2002), la montagne a acquis une visibilité inconnue jusqu'alors sur la scène internationale.

Ce résultat est le fruit des efforts combinés d'acteurs très différents:

- Les agences de l'Organisation des Nations Unies (ONU), notamment celles qui sont en charge de la promotion de politiques de développement durable: l'Organisation des Nations Unies pour l'alimentation et l'agriculture (FAO), le Programme des Nations Unies pour l'environnement (PNUE), l'Organisation des Nations Unies pour l'éducation, la science et la culture (UNESCO).

- Quelques Etats décidés à jouer cette carte sur la scène internationale, notamment la Suisse, qui a fait de la montagne l'objet emblématique de ses actions de coopération (DIRECTION DU DÉvELOPPEMENT ET DE LA COOPÉRATION 2002) et un des instruments stratégiques de son positionnement à l'ONU et dans ses agences.

- Des associations et institutions diverses émanant des milieux scientifiques, des organisations écologistes, des institutions régionales, voire des associations d'habitants, qui souhaitaient être associées à la réflexion.

L'identification de la montagne comme enjeu planétaire n'est donc pas le fruit de la seule coopération entre Etats dans le cadre des organisations internationales. Elle est le fruit d'une interaction complexe entre des protagonistes de nature, de spécialités et d'échelles d'intervention très différentes. C'est en raison de ce mode de gestation inhabituel, mais aussi en raison du souci de plus en plus clairement affiché par l'ONU et plus spécialement par sa Commission du développement durable, d'associer des acteurs de terrain et les populations à ses actions (NATIONs UNIES 2003) que la principale scène de réflexion et de coordination, qui a vu le jour dans le cadre de ce processus, a pris la forme d'un «Partenariat international pour le développement durable des régions de montagne» associant des acteurs de nature très diverse (p.ex. Etats, organisations intergouvernementales, organisations non gouvernementales, entreprises, équipes de recherche).

Une préoccupation comparable relative à la gestion des environnements montagnards a conduit à des accords internationaux à l'échelle de massifs de montagne et à l'implication d'acteurs très hétérogènes dans les processus de mise en ouvre. Les Alpes ont servi de laboratoire en la matière avec la conception, l'adoption, puis la mise en ouvre de la Convention alpine (1991) par les Etats alpins et l'Union européenne (UE) qui s'accordaient alors à promouvoir un développement maîtrisé de l'espace alpin. Bien que de nature strictement intergouvernementale, elle a été promue par des acteurs divers (notamment des organisations écologistes) et a suscité la création et l'implication d'institutions variées, notamment des gestionnaires d'espaces protégés (Réseau Alpin des Espaces Protégés) et des communes soucieuses de promouvoir un développement durable à leur échelle (Alliance dans les Alpes). Des initiatives comparables ont été mises en œuvre ou sont en train de l'être dans d'autres massifs de montagne (Carpates, Caucase, Pyrénées, Asie Centrale), souvent sur le mode de la coopération entre régions de montagne (Ministère ALlEMAND DE L'ENVIRONNEMENT, DE LA PROTECTION DE LA NATURE ET DE LA SÉCURITÉ NUCLÉAIRE 2004).

A l'échelle planétaire comme à l'échelle régionale, on observe donc un intérêt croissant pour une gestion internationale et transnationale des régions et des milieux de montagne. Dans les deux cas, le souci d'associer des acteurs à toutes les échelles, notamment locale, et issus de milieux très différents est manifeste. Dans ce contexte, l'objectif de cet article est d'étudier un ensemble de partenariats à distance construits par des institutions et des groupes locaux et de déterminer ce qu'ils doivent à ce processus de mondialisation et de régionalisation des enjeux montagnards et, symétriquement, ce qu'ils doivent à d'autres formes de motivation. L'exemple retenu est celui des régions de montagne de Suisse. 


\section{Tendances et analyses des recompositions institu- tionnelles et identitaires au temps de la mondialisa- tion}

L'objectif de cet article ainsi formulé renvoie à divers types d'analyses politiques, économiques et culturelles du processus de mondialisation et de régionalisation.

Un premier groupe de publications portent sur les transformations récentes connues par les modes de régulation territoriale des Etats-nations. Ces derniers sont soumis à deux tendances générales: d'une part une tendance à la décentralisation et à la régionalisation des institutions; d'autre part une montée en puissance de la coopération internationale, sinon de l'intégration supra-nationale (BADIE 1995; TAYLOR 1985) Sous l'effet de ces deux tendances, les administrations nationales ont été incitées à développer des formes de coordination des actions publiques à l'échelle internationale, mais aussi entre elles-mêmes et les administrations de niveau inférieur. Dans ce contexte, il conviendrait de déterminer ici, une fois évaluée l'ampleur de ce phénomène de partenariats à distance pour les régions retenues, dans quelle mesure les partenariats à distance entre communes de montagne rendent compte de ces tendances et, le cas échéant, relèvent de ce souci de coordination multi-échelles.

Un deuxième groupe de travaux, très hétérogène au demeurant, s'est intéressé au rôle croissant des préoccupations identitaires dans les recompositions sociales, les stratégies économiques et l'émergence de nouvelles formes d'action collective. De nombreux auteurs ont montré que les nouvelles conditions de circulation des hommes et des informations avaient permis la construction et l'expression d'identités transnationales (Appadurai 1996; Featherstone et al. 1995); d'autres ont souligné la résurgence des identités locales et régionales, notamment par l'entremise d'une exaltation de caractères singuliers empruntés à l'environnement ou à des faits de culture, ces identités devenant parfois des motifs d'action collective plus ou moins contestataires (Castells 1999; Poletta \& Jaspers 2001); d'autres enfin ont montré que ces préoccupations identitaires trouvaient parfois à s'investir dans des stratégies de développement économique sous la forme de valorisation symbolique de quelques attributs spécifiques des sociétés et des territoires locaux (Pecqueur 2004; SAVY \& Veltz 1995; Veltz 1996). Tous ensemble, ces auteurs ont bien montré que les recompositions identitaires actuellement à l'œuvre dans les sociétés contemporaines avaient des formes d'expression variées et suscitaient des modalités d'action tout aussi diverses.

Dans ce contexte, on peut se demander dans quelle mesure les sociétés vivant dans les montagnes suisses se servent de partenariats à distance pour exalter des formes d'identité traditionnelles ou émergentes, ou pour les opérationnaliser dans des projets d'action ou de développement spécifiquement tournés vers une valorisation de leur caractère montagnard.

Bien qu'elles portent ici spécifiquement sur les initiatives locales en Suisse, ces questions trouvent quelques éléments de comparaison avec d'autres contextes et d'autres échelles. Bien que peu de travaux synthétiques pour l'ensemble de ce type de régions soient disponibles (Debarbieux \& Gillet 2001), de nombreuses observations partielles soulignent que la référence à la montagne a motivé ou justifié des initiatives de cet ordre: organisation de lobbies tels que des associations d'élus (GERBaux 2004); constitution de réseaux d'échanges régionaux et planétaires comme le Mountain Forum; constitution de filières de production et de commercialisation des productions dites de montagne (Euromontana 2004); le tout dans un contexte d'initiatives intergouvernementales et transnationales nombreuses. Bien que relevant de domaines très différents, ces exemples soulignent chacun à leur manière la propension croissante à identifier des lieux et des individus à un caractère montagnard, et à faire reposer une stratégie d'action (p.ex. politique, économique, culturelle) sur cette identification.

Cet article vise donc à prendre la mesure de ces partenariats montagnards à l'échelle des communes suisses et à déterminer dans quelle mesure ils participent des phénomènes de recompositions institutionnelles et identitaires évoquées ci-dessus.

\section{Dénombrement des partenariats entre communes de montagne}

L'identification des partenariats à distance construits par des collectifs et des institutions locales a été réalisée à l'aide d'un très bref questionnaire adressé aux administrations communales. Certes, seule une partie des partenariats visés par cet article sont initiés et portés par les communes elles-mêmes; mais compte tenu de la légitimité acquise de longue date et de la tradition démocratique de cet échelon territorial, il apparaissait le plus à même de disposer de l'information recherchée.

Les administrations communales auxquelles ce questionnaire a été adressé sont toutes celles qui sont concernées par l'un ou l'autre des textes officiels définissant la montagne à l'échelle de la Confédération (Loi fédérale sur l'aide aux investissements dans les régions de montagne et Ordonnance sur le cadastre de la production agricole et la délimitation de zones), soit 1453 communes. 1132 réponses ont été recueillies, soit un taux de retour de près de $78 \%$. Les informations collectées ont été recoupées avec celles mises à disposition par plusieurs réseaux tels que l'Association suisse 
pour le Conseil des Communes et Régions d'Europe ou l'Alliance dans les Alpes, ainsi que par la Direction du développement et de la coopération suisse (DDC). Ce recoupement des informations recueillies a permis d'identifier 413 communes concernées par ce genre de partenariats et un total de 578 initiatives, ce qui atteste, bien que ce dénombrement ne soit pas exhaustif, de la vigueur de ce type d'échanges en Suisse.

Une seconde étape a consisté à déterminer le caractère montagnard des communes étrangères impliquées dans ces partenariats. Pour les territoires situés au sein de l'UE, soit environ $85 \%$ des partenariats recensés, le travail d'identification s'est appuyé sur la définition adoptée par une étude de la Commission européenne (NoRDREGIO 2004). Pour les communes restantes, situées hors de l'UE, le dénombrement s'est appuyé, quoique de façon informelle, sur les critères adoptés par le PNUE dans sa définition des régions de montagne à travers le monde (KAPOs et al. 2000), critères au demeurant très proches de ceux adoptés dans l'étude européenne susmentionnée. Cette étape du travail a montré qu'environ $56 \%$ des communes ayant identifié des partenariats (231 sur 413 ) et environ $48 \%$ des partenariats eux-mêmes (277 sur 578) mettait en relation des populations vivant en montagne selon les définitions utilisées.

Un deuxième questionnaire a été adressé aux seules communes suisses de montagne qui entretenaient des partenariats avec des communes de montagne situées ailleurs dans le monde. Il s'agissait alors d'obtenir des précisions sur le contenu des partenariats, leur origine, les motivations qui les sous-tendaient et d'identifier ceux pour lesquels le caractère montagnard des communes impliquées était explicitement invoqué: ce type de motivations concerne 151 communes suisses et 170 partenariats. Le premier résultat auquel conduit cette recherche est donc le suivant: environ un tiers des communes suisses de montagne pour lesquelles des partenariats à distance ont été identifiés les ont construits avec des communes situées dans d'autres régions de montagne du monde avec un objectif explicite de travailler sur une ou plusieurs questions relatives à leur environnement montagnard ou à un mode de vie ou de production conçu comme montagnard (Fig. 1).

En outre, 53 initiateurs et porteurs de partenariats ont été interrogés de façon approfondie. Ces entretiens semidirectifs visaient à recueillir des faits et des interprétations sur la genèse, les motivations et les modalités de ces actions. Enfin quelques informations ont été recueillies de façon ponctuelle, sans pour autant qu'elles présentent de valeur comparable pour la méthode exposée: entretiens de responsables d'échanges de ce type à Aspen et Vail (Etats-Unis), participation à deux manifestations culturelles organisées dans le cadre de ce genre de partenariat et présence à une réunion d'association.

\section{Typologie des formes de partenariats}

Condition première de la constitution du corpus, tous les partenariats retenus pour cette recherche reposent sur l'idée que les communes concernées partagent un attribut ou un caractère montagnard, attesté ou imaginé, qui justifie la conduite d'un projet commun. Mais ces partenariats s'avèrent très divers dans la forme et dans l'esprit. Une typologie analytique a été construite en fonction de trois critères qui découlent des questions posées dans cette recherche:

- Les objectifs affichés: puisque cette recherche pose l'hypothèse que des préoccupations identitaires motivent ces partenariats, il convenait d'identifier la nature exacte et le poids relatif de ce type de motivations, les façons que l'on avait d'invoquer des identités montagnardes et la nature politique, économique et culturelle des projets au service desquels elles étaient mises.

- La présence ou l'absence d'un pilotage ou d'une coordination nationale ou supra-nationale: ce critère semble le plus à même d'évaluer le souci de coordination multi-échelles des initiatives relatives à la montagne dont on a dit plus haut qu'il était très présent aussi bien dans les déclarations des organisations et des partenariats internationaux que dans celles des administrations fédérales concernées.

- Le nombre de partenaires: ce critère est apparu utile pour prendre la mesure du poids qu'un réseau ou un partenariat était capable d'acquérir dans ses relations avec d'autres institutions.

Le traitement des questionnaires et des entretiens, par analyse de contenu, à l'aide de cette combinaison de critères a permis de dégager cinq types principaux de partenariats. Ils sont successivement présentés ici, chacun étant illustré à l'aide d'un exemple représentatif.

\subsection{Type 1: réseaux d'échange et lobbies}

Le premier type de partenariat identifié est celui du large réseau d'échange entre communes qui vise autant à l'échange d'informations et de savoir-faire qu'à constituer un partenaire de poids dans un système d'institutions. Une association, Alliance dans les Alpes, constitue le seul cas de ce type pour les communes étudiées.

Quelques années après la signature de la Convention alpine, la Commission internationale pour la protection des Alpes a encouragé des communes volontaires à s'associer pour mettre en ouvre à leur échelle des politiques de développement durable dans les domaines jugés prioritaires (p.ex. agriculture, forêt, sol, énergie, nature et paysage, aménagement du territoire, tourisme). A cette fin, elle a mis sur pied un réseau de communes, intitulé Alliance dans les Alpes, dont la mission principale est de favoriser l'échange d'informations et d'expériences au travers de l'organisation 


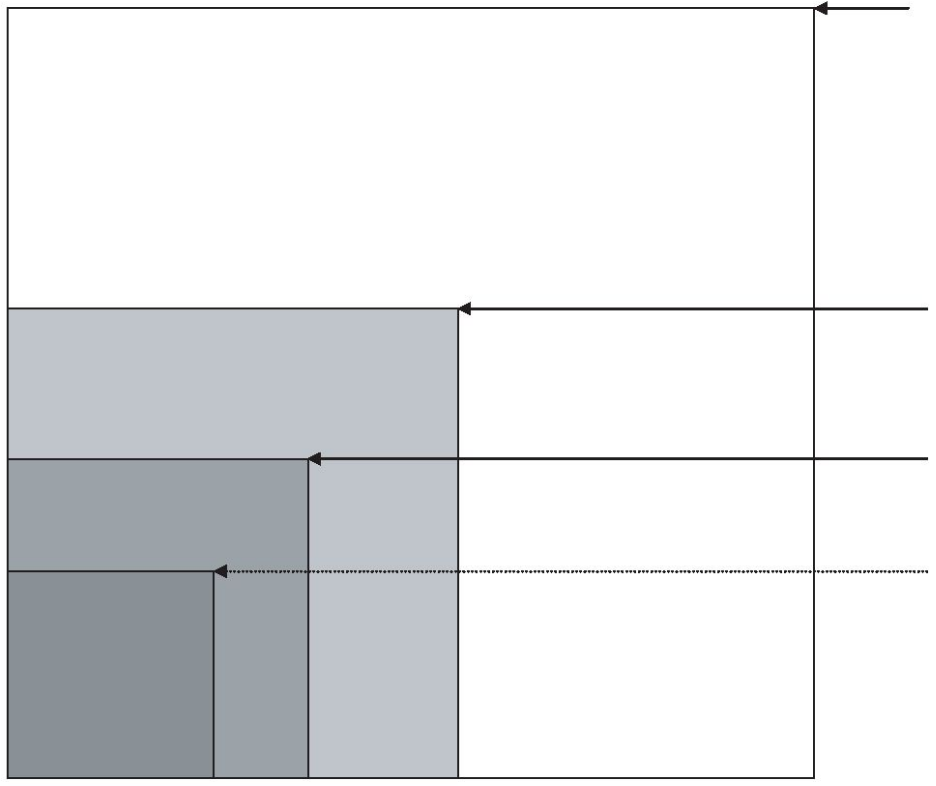

Communes suisses ayant tout ou partie de leur territoire en zone de montagne selon la législation suisse $(n=1453)$

Communes ayant mentionné au moins un partenariat à distance $(n=413)$

Partenariats avec une commune étrangère située en montagne $(n=231)$

Partenariats motivés par un attribut montagnard ou une qualité considérée comme montagnarde $(\mathrm{n}=151)$

Fig. 1: Dénombrement des partenariats

Aufzählung der Partnerschaften

Partnerships enumeration

d'ateliers thématiques et de visites de sites. Le réseau, fondé en 1997 par 27 communes, compte actuellement près de 200 membres, dont 95 en Suisse.

Motivé par des considérations pratiques, ce réseau transnational répondait aussi à un souci politique. Il s'agissait pour ces communes de se présenter comme un interlocuteur légitime vis-à-vis des protagonistes de la gestion de l'espace alpin, Alliance dans les Alpes offrant

«la possibilité de faire entendre sa voix dans le concert des communes alpines au sein de la future Europe des régions» (Alliance dans les Alpes 2002: 6).

Cette association affichait donc pour objectif de peser sur les Etats signataires, dont la Suisse, et sur l'UE pour qu'ils approfondissent leur coopération alpine et qu'ils engagent des actions concrètes conformes aux objectifs de développement durable des Alpes. A cette fin, l'association s'est organisée en sections nationales qui s'efforcent d'apparaître comme des partenaires naturels de toute réflexion institutionnelle sur les Alpes.

\subsection{Type 2: jumelages Est-Ouest}

Un second type de partenariat correspond aux échanges entre communes de différents pays d'Europe au titre de la promotion, par les Etats correspondants, de la paix et de la compréhension mutuelle à l'échelle du continent. Une première génération de ce type d'échanges, qui a souvent pris la forme de jumelages, a concerné des communes d'Europe de l'Ouest au lendemain de la Seconde Guerre mondiale. La chute des régimes communistes à partir de 1989 a permis d'étendre cette forme de coopération à l'Europe centrale et orientale, notamment dans le but de promouvoir des modes de gouvernement et de gouvernance démocratiques à l'échelle locale. En Suisse, plusieurs initiatives de grande ampleur, avec la Roumanie («Opération villages roumains») et la Tchéquie notamment, ont mobilisé un nombre important de communes, mais sans que les communes de montagne soient particulièrement ciblées.

En revanche, l'association Causes Communes Suisse est intervenue en ex-Yougoslavie, en associant notamment les communes neuchâteloises de La Chaux-de-Fonds et du Locle. Par ailleurs, plusieurs communes de l'Oberland bernois ont noué des partenariats avec des communes bulgares de la Stara Planina, répondant ainsi aux encouragements de l'association Inter-Assist, un réseau d'experts suisses promouvant l'aide au développement dans les régions de montagne. Si les premières actions d'Inter-Assist visaient à faire coopérer des hôpitaux suisses et bulgares, un objectif plus politique a guidé les opérations ultérieures: les communes bernoises ont été invitées à aider les communes bulgares dans la réforme de leur administration et de leurs modes de gouvernement local (institutional building). Les entretiens approfondis ont montré que pour ces deux familles d'opérations, l'implication de communes partenaires reposait sur des similitudes supposées, notamment une référence partagée à l'image du «libre montagnard» plusieurs fois mentionnée dans les entretiens. 


\subsection{Type 3: coopération décentralisée et aide au développement}

Un troisième type d'initiatives relève de ce que l'on appelle généralement la coopération décentralisée. Il implique des communes et des associations situées en Suisse et dans des pays du Sud, soucieuses de promouvoir des échanges culturels et des politiques locales de développement. Mais il implique aussi l'administration fédérale, à travers la DDC, en guise de coordonnateur, parfois initiateur, souvent contributeur financier.

Ainsi les régions de l'Obersimmental (Berne) et de Kotschkor (Kirghizstan) coopèrent dans le maintien de l'agriculture de montagne et la promotion d'activités économiques complémentaires (tourisme doux, vente de tapis kirghizes); une association de l'Oberland bernois a noué des contacts comparables avec l'ethnie Kalash (Pakistan); des échanges ont été mis sur pied à partir de 2000 entre des paysannes suisses de montagne et des paysannes ladakhis (Inde) dans le but d'échanger des expériences, des compétences et des connaissances dans le domaine agricole; enfin, en 2002, une coopération entre Valaisans et Népalais a débouché sur la valorisation des savoir-faire des habitants de ces deux régions en matière d'irrigation des versants.

Dans ce type d'opérations, les bénéfices attendus visent autant les populations du Sud que celles de Suisse. Comme le résume l'initiatrice du partenariat entre paysannes suisses et femmes ladakhis, ces échanges permettent à chacun des groupes concernés de développer «un meilleur sentiment de sa propre valeur» (Roselu 2004: 15). La charte du partenariat Obersimmental-Kotschkor signée par les instances régionales affiche l'ambition de défendre les régions de montagnes comme espace de vie et comme espace économique. L'action de partenariat permet de délivrer un message positif sur les sociétés concernées:

«Les deux régions de montagne doivent constamment se faire entendre et faire remarquer l'indispensable valeur des régions de montagne» (Alpverein Obersimmental 2002).

Le point commun de ces opérations est qu'elles reposent essentiellement sur les initiatives de personnes très impliquées pour des raisons professionnelles ou dans des contextes associatifs dans le développement des pays du Sud et les échanges culturels. Des organisations de coopération sont impliquées dans ces initiatives à l'instar de la DDC pour les partenariats avec Kotschkor, Kalash et Ladakh et l'organisation non gouvernementale Helvetas pour le Népal. Les communes jouent ici un rôle moindre que dans les autres formes de partenariat, dans le meilleur des cas un rôle d'accompagnement d'un projet qu'elles n'ont pas initié elles-mêmes.

\subsection{Type 4: clubs économiques et partenariats entre stations de skis}

Un quatrième type de partenariat répond à des objectifs économiques et relève presque exclusivement de l'initiative des acteurs locaux. Il s'agit principalement de communes et d'offices du tourisme de stations et de sites touristiques. Ces derniers, principalement motivés par une recherche de plus-value économique, obéissent à une logique de «club», qui rassemble des lieux renommés et les conforte dans leur position de destination privilégiée en se distinguant d'autres stations.

L'archétype de ce type de partenariat est le réseau intitule Best of the Alps qui rassemble douze stations touristiques majeures des Alpes, dont quatre suisses (Davos, Grindelwald, St.Moritz et Zermatt) autour d'une plate-forme marketing commune mise sur pied à la fin des années 1980 pour approcher les marchés internationaux. Quoique moins fédérateurs, des partenariats bilatéraux ont également été construits pour établir des liens durables avec des sites touristiques plus lointains, comme entre Saas Fee et Steamboat Springs (Etats-Unis); Grindelwald et Azumi-Mura (Japon); Davos et Aspen (Etats-Unis); Chamonix (France) et Sanada (Japon); Zermatt, Myoko Kogen (Japon) et Sexten (Italie). L'objectif affiché alors est de favoriser l'échange d'expériences entre sites touristiques et entre stations de ski d'importance, de notoriété et de standing comparables.

Les stations américaines et asiatiques impliquées dans un partenariat de ce type cherchent à bénéficier de la notoriété de leurs partenaires européens et de l'image montagnarde de la Suisse. Pour le partenaire suisse, le partenariat s'inscrit souvent dans une démarche de promotion touristique. Le choix stratégique des partenaires est révélateur de cet objectif. Ainsi, par exemple, avec l'émergence du marché chinois, perçu comme étant prometteur, un jumelage a été noué entre la région du Cervin et celle de Yulong Snow Mountain et un autre entre la région de la Jungfrau et celle du Mont Huangshan, qui bénéficient toutes deux du statut de site du Patrimoine mondial de l'humanité. Pour ces deux derniers partenariats, l'implication de Suisse Tourisme atteste de la plus-value économique recherchée.

\subsection{Type 5: promotion des identités culturelles au cas par cas}

Un dernier type de partenariats correspond à des initiatives circonstancielles d'acteurs locaux qui visent essentiellement à promouvoir des échanges culturels. A Sainte-Croix (Vaud), l'Association Gens des Hauts Pays organise tous les deux ans une manifestation qui permet la rencontre des gens du Balcon du Jura avec une autre communauté montagnarde (village d'Aspindza en Géorgie en 2003; deux communes du Pays basque français en 2005) autour de festivités musicales 
et gastronomiques. Dans un objectif comparable, les communes de Claro (Tessin) et Valle di Cadore (Italie) organisent depuis 2002 des rencontres d'habitants et d'écoliers.

Le souci de s'ouvrir aux montagnes du monde est manifeste dans ces initiatives. Mais ce type d'initiative vise aussi à rassembler les habitants des communes suisses autour d'un projet identitaire local. L'Association Gens des Hauts Pays affiche ainsi l'objectif d'associer les habitants à un projet collectif censé renverser l'image de leur commune, altérée par la crise industrielle, et, pour ce faire, de «fédérer les forces associatives existantes sur le Balcon du Jura» (Association Gens des Hauts Pays 2005). A Claro, les entretiens ont montré le souci de développer un attachement des habitants au lieu de leur résidence. Pour le vice-maire la mise en place d'un partenariat lointain permet de créer «une occasion de rencontre» entre anciens et nouveaux habitants de la commune. L'ouverture des communes de montagnes suisses à d'autres communautés de montagne dans le monde est donc conçue comme une façon de renforcer les identités locales et de tisser du lien social à cette échelle.

\section{Conclusion}

L'inventaire des partenariats à distance construits par des communes suisses des Alpes et du Jura montre la forte propension des acteurs locaux à nouer des contacts avec des populations de montagne situées ailleurs dans le monde et à mettre en avant, au travers de ces contacts, leur caractère montagnard. Mais ce souci commun d'échanger «entre montagnards» recouvre une très grande diversité de formes et d'objectifs visés, et une grande variété dans les façons de travailler les identités montagnardes. Il s'agit tantôt d'opérations de promotion économique dans lesquelles les images ou les identités de produits priment sur les identités collectives, tantôt de construction de lobbies, où l'identité collective est mise au service d'un projet politique, tantôt d'une célébration des cultures montagnardes, et souvent d'une combinaisons de motivations hétérogènes. Ce ne sont donc pas les mêmes façons de se penser «montagnard» qui sont mises en œuvre.

Rapportées aux initiatives de la Suisse sur la scène internationale, ces partenariats ont souvent été conçus en rapport avec elles et non comme des manifestations d'autonomie ou de défiance. En effet, ces partenariats s'avèrent très complémentaires aux initiatives de la DDC ou à celles de Suisse Tourisme qui invoquent souvent les mêmes imaginaires de la montagne et les mêmes savoir-faire montagnards. Les communes suisses concernées s'inscrivent donc dans des projets complémentaires à ceux de la Confédération, mais aussi à ceux de plusieurs cantons. Ici, les échanges transnationaux mis sur pied par les communes et acteurs des montagnes suisses apparaissent comme un engagement des sociétés locales dans une déclinaison des imaginaires helvétiques, autrefois fondateurs de l'identité nationale et régionale, aujourd'hui déployés à l'échelle du monde. La seule exception, relative, à ce caractère commun est représentée par Alliance dans les Alpes dont l'un des objectifs est de pousser la Confédération à être plus volontaire dans la promotion du développement durable à l'échelle alpine.

En revanche, ces identités en action, très hétérogènes de nature et visant des objectifs divers, ne reflètent que faiblement les initiatives internationales autour de la montagne, quelques communes et acteurs seulement y faisant explicitement référence, et l'Année Internationale de la Montagne n'ayant manifestement suscité que quelques rares opérations.

\section{Bibliographie}

Alliance dans les Alpes (2002): Rapport 1997-2002, les 5 premières années du réseau Alliance dans les Alpes. - Mäder: Alliance dans les Alpes.

Alpyerein Obersimmental (2002): Partnerschaft Kotschkor-Obersimmental, gemeinsame Erklärung. - Lenk: Obersimmental Alpverein.

Appadurai, A. (1996): Modernity at large. Cultural dimensions of globalization. - Minneapolis: University of Minnesota Press.

Association Gens des Hauts Pays (2005): Quand les peuples de montagne se rencontrent. - Sainte-Croix: Association Gens des Hauts Pays.

BADIE, B. (1995): La fin des territoires. - Paris: Fayard. Castells, M. (1999): Le pouvoir de l'identité. - Paris: Fayard.

Debarbieux, B. \& F. Gillet (2001): La montagne: un objet de recherche $?-=$ Revue de géographie alpine 2 . DIRECTION DU DÉVELOPPEMENT ET DE LA COOPÉRATION (2002): Les montagnes et leurs habitants: un compterendu des programmes de développement des régions de montagne soutenus par la Direction du développement et de la coopération. - Berne: Direction du développement et de la coopération.

Euromontana (2004): Produits agroalimentaires de montagne en Europe. - Bruxelles: Euromontana.

Featherstone, M., Lash, S. \& R. Robertson (1995) Global modernities. - London: Sage.

Gerbaux, F. (2004): Les groupes de pression et la défense des zones de montagne européenne. - In: Revue de géographie alpine 2: 17-26.

Kapos, V., Rhind, J., Edwards, M.-F., Price, M. \& C. Ravilious (2000): Developing a map of the world's mountain forests. - In: PrICE, M. \& N. ButT (éds): Forests in sustainable mountain development. - Wallingford: CABI: 4-9. 
MiNistère ALLEMAND DE L'ENVIRONNEMENT, DE LA PROTECTION DE LA NATURE ET DE LA SÉCURITÉ NUCLÉAIRE (2004): International partnerships for sustainable development in mountain regions. Mountain partnerships under the Alpine Convention. - = Umwelt 3, 02.2004, Berlin.

Messerli, B. \& T. Hofer (2003): Von der Welt der Berner Alpen zu den Gebirgen der Welt. - In: JEANNERet, F., Wastl-Walter, D., Wiesmann, U. \& M. Schwyn (éds): Welt der Alpen - Gebirge der Welt: Ressourcen, Akteure, Perspektiven. - Bern: Haupt: 9-21.

Nations Unies (2003): UN system and civil society. An inventory and analysis of practices. - http://www. un.org/reform/pdfs/hlp9.htm 30.05.2006.

NordRegio (2004): Zones de montagne en Europe. Analyse des régions de montagne dans les Etats membres actuels, les nouveaux Etats membres et d'autres pays européens. - Bruxelles: Direction générale de la politique régionale - Commission européenne.

Pecqueur, B. (2004): Vers une géographie économique et culturelle autour de la notion de territoire. - In: Géographie et culture 49: 22-37.

Poletta, F. \& M. Jaspers (2001): Collective identities and social movements. - In: Annual review of socio$\operatorname{logy} 27: 283-305$.

Roselli, M. (2004): Willisau-Ladakh: la filière de la fraise. - In: Un seul monde 1, 03.2004, Berne: 14-15. Savy, M. \& P. Veltz (1995): Economie globale et réinvention du local. - La Tour d'Aigues: Editions de l'Aube/DATAR.

TAYLOR, P. (1985): Political geography, world-economy. Nation-state and locality. - Londres: Longmans.

VeLTz, P. (1996): Mondialisation, villes et territoires. - Paris: Presses Universitaires de France.

\section{Résumé: Des communes suisses en réseau. La réfé- rence à la montagne dans les partenariats à distance noués par les acteurs locaux}

Comme un grand nombre d'autres types de régions et de milieux, les montagnes sont concernées par le rôle croissant des initiatives internationales et transnationales. Si les initiatives intergouvernementales sont relativement bien connues, il n'en est pas de même des formes de coopération et des réseaux initiés par les acteurs locaux. Cet article s'intéresse spécifiquement aux partenariats à distance construits par des communes suisses de montagne avec d'autres communautés de montagne à travers le monde. Un état de la situation rappelle l'importance quantitative de ces partenariats et le rôle de l'exaltation des attributs et des valeurs associés à la montagne. L'analyse montre que les motivations de ces partenariats combinent des préoccupations politiques, culturelles et économiques et que les partenariats conduits par les communes sont, dans l'ensemble, complémentaires aux initiatives de la Confédération et des cantons, en mobilisant des imaginaires similaires de la montagne.

\section{Zusammenfassung: Vernetzte Schweizer Gemeinden. Internationale Partnerschaften lokaler Akteure und die Bedeutung der Berge}

Wie viele andere Regions- und Milieu-Typen sind Berggebiete von der zunehmenden Präsenz der internationalen und transnationalen Initiativen betroffen. Während die entsprechenden Initiativen unter Staaten relativ gut bekannt sind, ist dies bei Zusammenarbeitsformen und Netzwerken von lokalen Akteuren weniger der Fall. Dieser Artikel diskutiert internationale Bergpartnerschaften, die Schweizer Berggemeinden mit anderen Berggemeinschaften weltweit aufbauen. Eine Gesamtaufnahme hat die quantitative Wichtigkeit dieser Partnerschaften und die Rolle der Begeisterung für die Eigenschaften und Werte, die mit dem Gebirge verbunden werden, aufgezeigt. Die Analyse zeigt, dass diese Partnerschaften politische kulturelle und wirtschaftliche Motivationen und Aufgaben miteinander verbinden, ferner, dass die durch die Gemeinden geführten Partnerschaften insgesamt komplementär zu denjenigen des Bundes und der Kantone funktionieren. Dabei mobilisieren sie ähnliche Vorstellungen in Bezug auf das Gebirge.

\section{Summary: Community networks in Switzerland. The importance of mountains in the international partner- ships of local stakeholders}

As in many other regions and milieus, mountainous areas are affected by the increasing presence of international and transnational initiatives. Although the workings of such initiatives at national level are well known, this cannot be said of the forms of collaboration adopted by local actors. This article focuses in particular on international mountain partnerships established by Swiss mountain communities. By means of a comprehensive survey, insight was gained into the quantitative value of such partnerships and the role of local enthusiasm for topographic-related characteristics and values. The analysis indicates that such partnerships allow political, cultural and economic motivation and activities to be combined. Furthermore, they appear to compliment federal and cantonal initiatives for these regions through the mobilisation of similar mountain images.

Prof. Dr. Bernard Debarbieux, Dr. Gilles Rudaz, Département de géographie, Faculté des Sciences économiques et sociales, Université de Genève, Boulevard du Pont d'Arve 40, CH-1211 Genève 4, Suisse.

e-mail: bernard.debarbieux@geo.unige.ch

e-mail: rudaz@berkeley.edu

Manuskripteingang/received/manuscrit entré le 30.5.2006

Annahme zum Druck/accepted for publication/accepté pour l'impression: 4.5.2007 